\title{
Theory and observation of double discontinuities
}

\author{
Y. C. Whang \\ Catholic University of America, Room 107, Pangborn Hall, Washington, D. C. 20064, USA
}

Received: 26 June 2003 - Revised: 1 December 2003 - Accepted: 2 December 2003 - Published: 14 April 2004

Part of Special Issue "International Workshops on Nonlinear Waves and Chaos in Space Plasmas"

\begin{abstract}
Recent research using high-resolution magnetic field data to examine the interior structures of MHD shocks in interplanetary space and in the magnetotail led to a surprising discovery that a slow-mode shock is often followed by an adjoining rotational discontinuity layer on the postshock side. The thickness of each layer is of the order of a few ion inertial lengths. Such a compound structure is known as a double discontinuity. When the magnetic field rotates by several degrees per ion inertial length inside a thin layer, the Hall current term becomes important in the generalized Ohm's law. Steady state solutions based on the Hall-MHD theory have been obtained to show the merging of a rotational layer and a slow shock layer to form a compound structure like the observed double discontinuities.
\end{abstract}

\section{Introduction}

MHD shock, rotational discontinuity, tangential discontinuity, and contact discontinuity are four kind of MHD discontinuities well understood in theory, and their existence in space plasma has been verified by using observational data from spacecraft. A small number of slow shocks have been observed in interplanetary space (Chao and Olbert, 1970; Burlaga and Chao, 1971; Richter et al., 1985; Whang et al., 1996) and in the magnetotail (Feldman et al., 1984, 1985, 1987; Smith et al., 1984; Seon et al., 1995; Saito et al., 1995, 1996).

Our recent research using high-resolution magnetic field data to examine the interior structures of MHD discontinuities in interplanetary space and in the magnetotail led to a surprising discovery that a slow shock is often followed by an adjoining rotational discontinuity layer on the postshock side. We call such a compound structure a double discontinuity (Whang et al., 1997, 1998, 2001).

Correspondence to: Y. C. Whang

(whang@cua.edu)
According to the ideal MHD theory, when a rotational discontinuity approaches a slow shock from the postshock side, the rotational discontinuity would always penetrate through the slow shock from behind and then continue to propagate away from the slow shock as a separate MHD discontinuity in the preshock region. Thus, the ideal MHD description cannot explain why the rotational discontinuity may not penetrate through the shock layer. The alternative is to explore whether the Hall-MHD theory can explain the merging of a slow shock layer and an adjoining rotational layer into a stable compound structure.

In this paper we first present the observational evidence for the compound structure composed of a slow shock layer and a rotational layer based on high-resolution data; the rotational layer has a thickness of the order of a few ion inertial lengths. Then we show that when the magnetic field rotates by several degrees per ion inertial length inside a thin layer, the Hall current term becomes important in the generalized Ohm's law. We show that steady-state Hall-MHD equations can produce solutions for compound structure composed of a slow shock and an adjoining rotational layer.

\section{Observation of double discontinuity in interplanetary space}

Typical identification of slow shocks from observational data is to treat the shock as a discontinuity and to analyze the jump conditions of the plasma and magnetic field on two sides of the shock. Figures 1 and 2 show an example for the observation of a forward slow shock in interplanetary space on 6 April 1995 from the Wind spacecraft. Observational data in Fig. 1, at the rate of one data point in every $84 \mathrm{~s}$, show the signature of a forward slow shock across which the proton density increases, the proton temperature increases, and the magnitude of magnetic field decreases. Available electron data from Wind have time resolution of $12 \mathrm{~s}$; Fig. 2 shows that the electron density has a sharp jump at the shock crossing. 


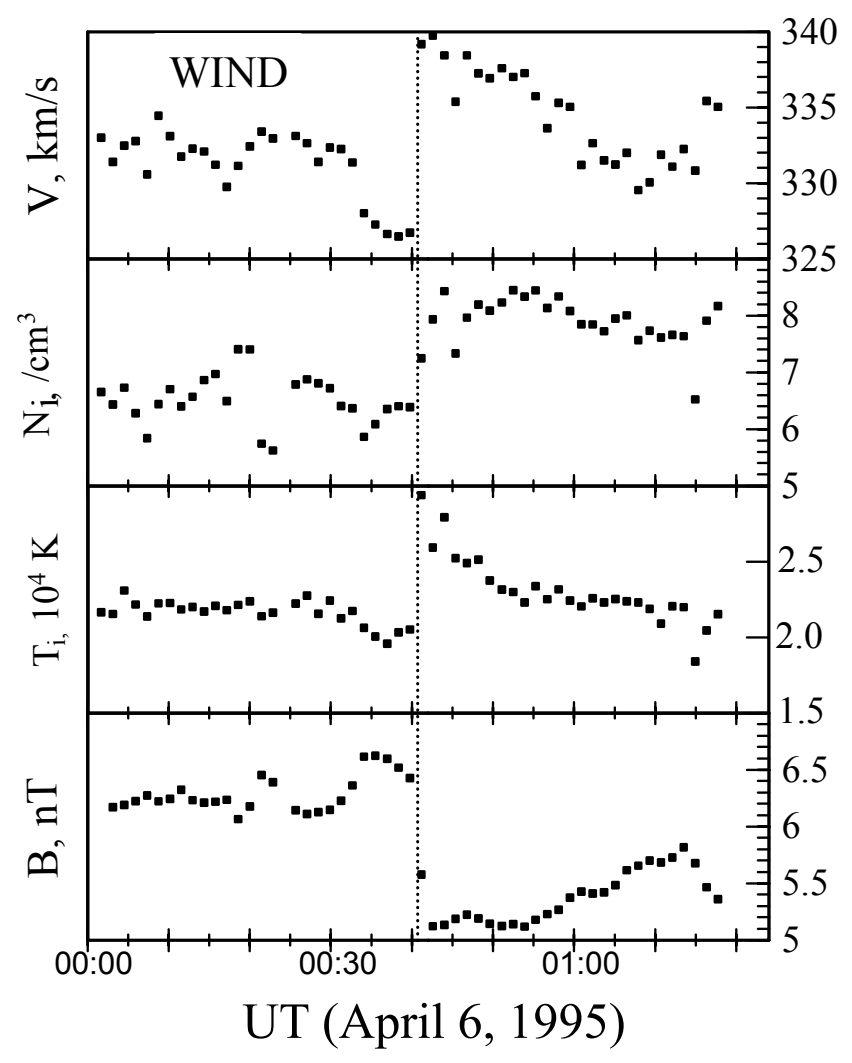

Fig. 1. Identification of a slow shock crossing on 6 April 1995, from the Wind magnetic field and proton data. The time resolution is $84 \mathrm{~s}$.

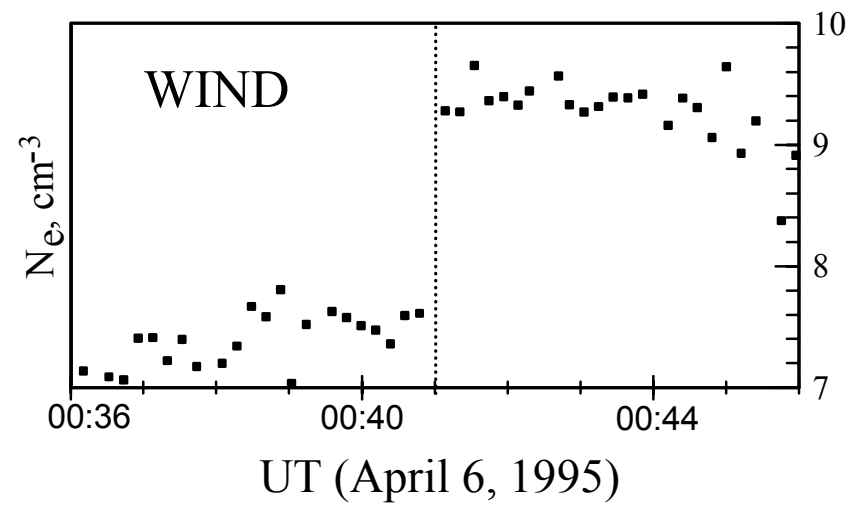

Fig. 2. The electron density has a sharp jump at the shock crossing. The time resolution of the electron data is $12 \mathrm{~s}$. If better timeresolution data are available, one can examine the internal structure of the shock.

Let subscripts $p$ and $e$ denote protons and electrons, subscripts 1 and 2 denote the preshock and the postshock conditions on both sides of the discontinuity, $U_{x}$ denote the normal components of the bulk velocity of the solar wind in the shock frame of reference, $\theta$ denote the shock angle (the angle between the shock normal and the magnetic field),
Table 1. Slow shock of the 6 April 1995 double discontinuities.

\begin{tabular}{lcc}
\hline Parameter & $\begin{array}{c}\text { Calculated from } \\
\text { observational data }\end{array}$ & $\begin{array}{c}\text { Rankine-Hugoniot } \\
\text { solutions }\end{array}$ \\
\hline \multicolumn{3}{c}{ Preshock conditions } \\
$\beta_{p 1}$ & 0.088 & \\
$\beta_{e 1}$ & 0.992 & \\
$\theta_{1}$ & $77.9^{\circ}$ & \\
$U_{1 x}(\mathrm{~km} / \mathrm{s})$ & 8.92 & \\
$U_{1 x} / a_{1} \cos \theta_{1}$ & 0.788 & \\
$U_{1 x} / C_{s}$ & 1.14 & \\
& & \\
$\theta_{2}$ & $76.0^{\circ}$ & \\
$U_{2 x}(\mathrm{~km} / \mathrm{s})$ & 7.05 & 0.724 \\
$U_{2 x} / a_{2} \cos \theta_{2}$ & 0.700 & 0.892 \\
$U_{2 x} / C_{s 2}$ & 0.882 & 0.807 \\
$B_{2} / B_{1}$ & 0.867 & 1.19 \\
$N_{2} / N_{1}$ & 1.27 & 1.12 \\
$\left(T_{p 2}+T_{e 2}\right) /\left(T_{p 1}+T_{e 1}\right)$ & 1.08 & \\
\hline
\end{tabular}

and $a$ and $C_{s}$ denote the Alfvén speed and slow speed. Table 1 show various shock parameters associated with the slow shock of 6 April 1995 derived from the observational data; the post shock conditions agree well with those calculated from the Rankine-Hugoniot solutions. The relative flow speeds $U_{x}<a \cos \theta$ on both sides of the shock, $U_{1 x}>C_{s 1}$ on the preshock side, and $U_{2 x}<C_{s 2}$ on the postshock side.

In the strict sense, MHD shocks are not discontinuities; a shock has a finite thickness across which physical properties change continuously. Observational data from the magnetic field, the proton, and the electron experiments are available at different time resolutions. The magnetic field data from Wind are available at the rate of one data point per $92 \mathrm{~ms}$. Making use of high-resolution data we can examine the interior structure within the finite thickness of the discontinuity observed on 6 April 1995.

In Fig. 3 we plot $12 \mathrm{~s}$ of high-resolution magnetic field data. Here the $x$ axis is the normal direction of the discontinuity surface pointing in the direction of the mass flow, let the $x, y$ plane be parallel to the magnetic field upstream of the shock layer,

$B_{t}^{2}=B_{y}^{2}+B_{z}^{2}$ and $\phi=\tan ^{-1}\left(B_{z} / B_{y}\right)$.

Then we can interpret the magnetic field structure of the observed discontinuity as a compound structure composed of two layers, a forward slow shock layer $S$ and an adjoining rotational layer $R$ on the postshock side. $B$ and $B_{t}$ decrease rapidly inside the $S$ layer. Across the $R$ layer the magnetic field rotates by an angle of $\sim 50^{\circ}$ about the normal direction of the shock surface, while its magnitude almost remains constant. The two successive layers are very close to each other, and hence the compound structure looks like a new kind of MHD discontinuity. The thickness of the rotational 


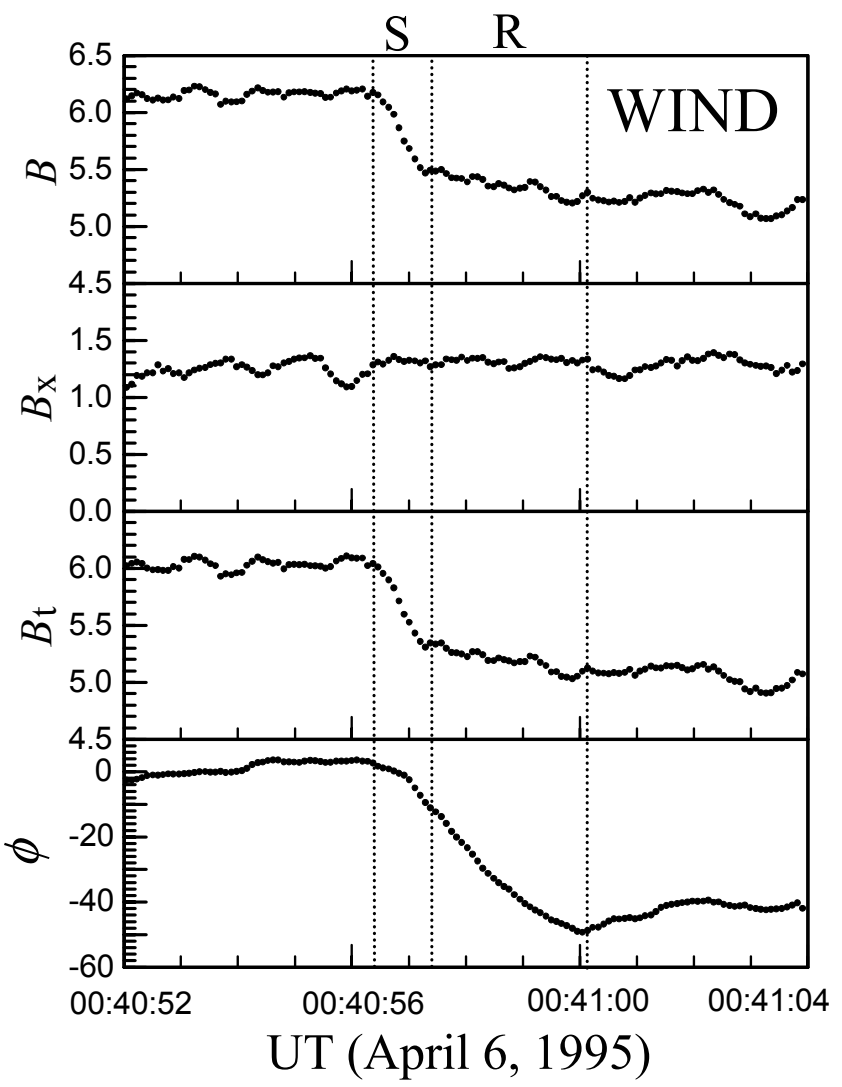

Fig. 3. The $12 \mathrm{~s}$ of high-resolution magnetic field data show the detailed structure of the shock discontinuity as a compound structure composed of an $S$ layer and an adjoining $R$ layer. The change in the magnitude of the magnetic field occurs mainly inside the $S$ layer, and across the $R$ layer the magnetic field rotates by an angle of $\sim 50^{\circ}$.

layer is of the order of 10 preshock ion inertial lengths, that is defined by $\lambda=c / \omega_{p i}$, where $\omega_{p i}=\left(4 \pi N_{1} e^{2} / m_{i}\right)^{1 / 2}$ is the ion plasma frequency, and $m_{i}$ the proton mass.

On 6 April 1995, the Geotail spacecraft was also upstream of the Earth's bow shock. The double discontinuity observed from Wind (Fig. 3) was also observed from Geotail (Fig. 4). The Geotail spacecraft crossed the double discontinuity at 51 min later than the crossing time of the Wind spacecraft. During this time interval the discontinuity surface has traveled $204 R_{E}\left(1.3 \times 10^{6} \mathrm{~km}\right)$ in interplanetary space, yet the double discontinuities observed from two spacecraft have nearly identical signatures. The two crossing points were at a distance of $\sim 60 R_{E}\left(0.4 \times 10^{6} \mathrm{~km}\right)$ on the surface of discontinuity. This means that the double discontinuity must be a reasonably stable solar wind structure and its size is larger than the dimension of the Earth's frontside magnetosphere.

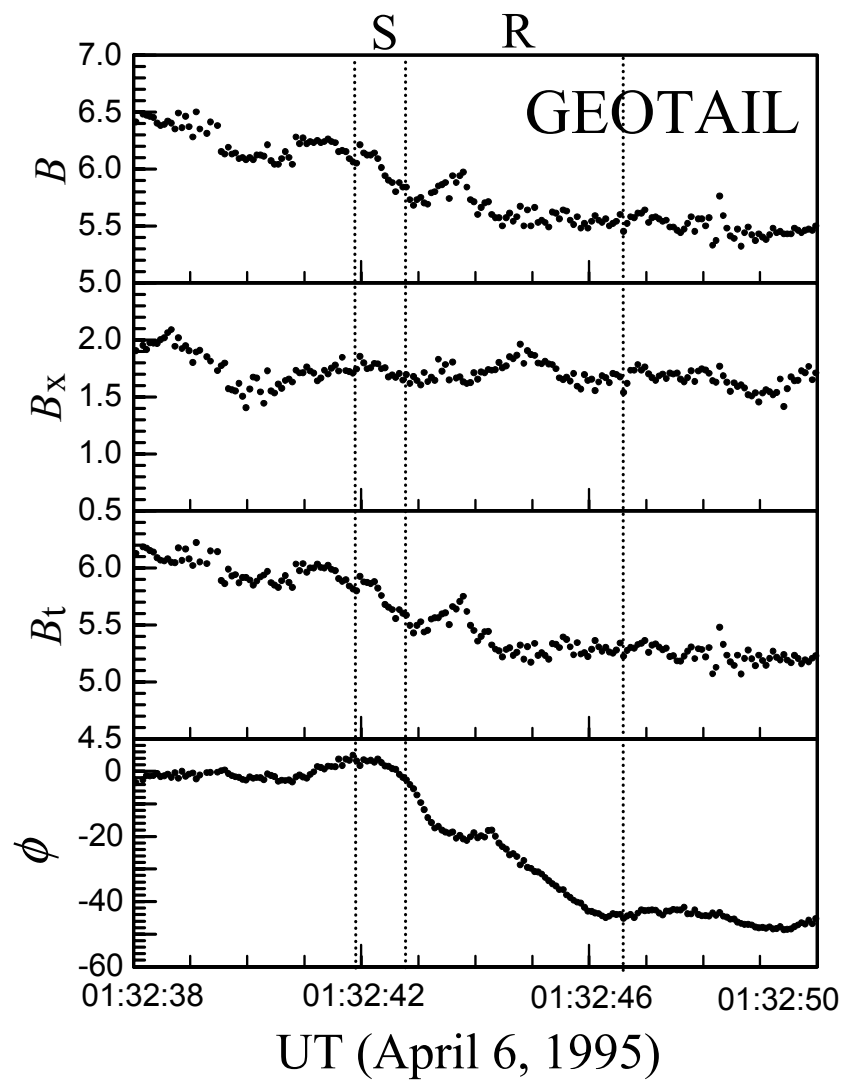

Fig. 4. The double discontinuity of 6 April 1995 was also observed from Geotail. The observed signature is nearly identical to the double discontinuities observed from Wind.

\section{Observation of double discontinuities in the magnetotail}

Double discontinuities exist not only in interplanetary space but also in the magnetotail region; they could be a general MHD structure in space plasma. Figure 5 shows $150 \mathrm{~s}$ of plasma and magnetic field data obtained from Geotail on 14 February 1994. The Geotail spacecraft crossed the boundary surface entering the plasma sheet from the north lobe of the magnetotail at $X_{\mathrm{GSM}}=-54 R_{E}$. The best available time resolutions of the magnetic field, the electron, and the ion data are, respectively $62.5 \mathrm{~ms}, 3 \mathrm{~s}$, and $12 \mathrm{~s}$. The magnetic field data play the vital part in identifying the rotational layer. The plasma density increases significantly and the magnetic field intensity decreases significantly across the interior of the slow shock layer ( $S$-layer). The $S$-layer is followed by an adjoining rotational layer (R-layer) on the postshock side. Across the $R$-layer the magnetic field rotates by $\sim 60^{\circ}$ about the normal direction of the shock surface, as the plasma density and the magnetic field intensity remain nearly unchanged.

The observed plasma and magnetic field signature is interpreted as the merging of a slow shock and a rotational discontinuity to form a stable compound structure. All observed 


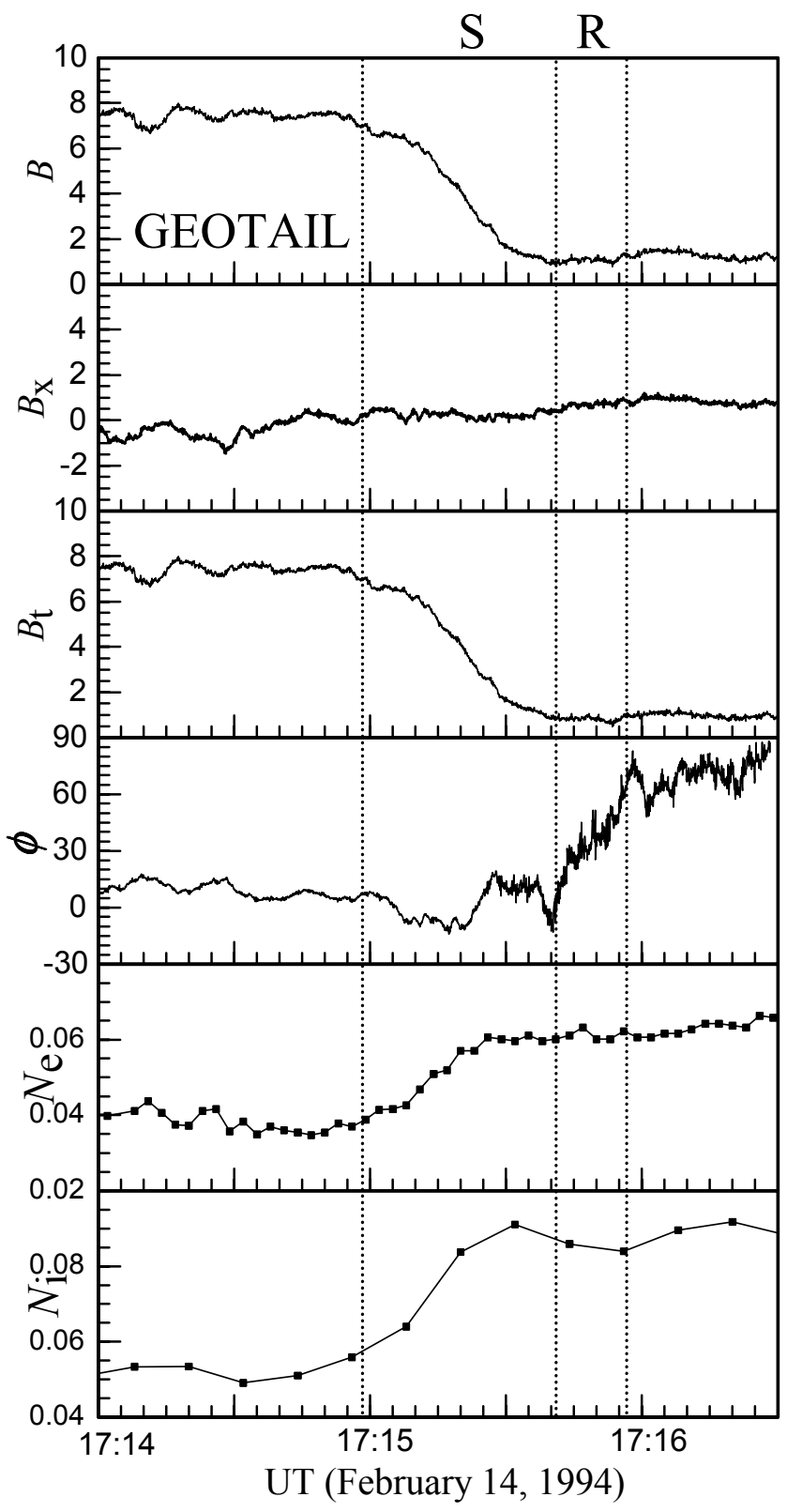

Fig. 5. A double discontinuity was observed on 14 February 1994 when the Geotail spacecraft crossed the boundary surface entering the plasma sheet from the north lobe of the magnetotail at $X_{\mathrm{GSM}}=-54 R_{E}$. Across the slow shock layer ( $S$-layer), the magnetic field intensity decreases and the plasma density increases. Through the adjoining rotational layer $(R$-layer) the magnetic field rotates by an angle of $\sim 60^{\circ}$ about the normal direction of the shock surface while the field intensity and plasma density remains nearly constant.

double discontinuities have large shock angles $\theta$ on the preshock side. The angle through which the magnetic field rotates inside the rotational layer can vary over a wide range; the rotation can be clockwise or counterclockwise. We must also point out that, in interplanetary space and in the magnetotail, a slow shock is not always followed by an adjoining rotational layer. We studied slow shocks and double discontinuities at the plasma sheet-lobe boundary using the Geotail data between 14 September 1993 and 16 February 1994 and the Wind data between 2 January 2000 and 5 January 2000, the occurrence ratio between the double discontinuity and the stand-alone slow shock is about 1.2.

\section{Steady-state Hall-MHD theory}

We interpret the formation of double discontinuity as resulting from the interaction of a rotational discontinuity approaching a slow shock from the postshock side; under certain conditions, the rotational discontinuity cannot penetrate through the shock and the two layers merge to form a stable compound structure. According to ideal MHD theory, after the interaction the rotational layer must penetrate through the slow shock layer; thus, the existence of such a compound structure cannot be explained based on ideal MHD theory.

Recently, Lee et al. (2000) studied whether plasma anisotropy is responsible for the existence of double discontinuity. They carried out a series of hybrid simulations to examine the interaction of a rotational discontinuity with a slow shock in anisotropic plasma. The simulation results cannot reproduce the formation of a double discontinuity.

We formulate the governing equation in the frame of reference attached to the discontinuity. The important dynamics of the problem is the rapid rotation of both the magnetic field and the fluid velocity inside a thin rotational layer. Inside the rotational layer the fluid velocity $\boldsymbol{u}$ is not field-aligned. As the magnetic field rotates through the rotational layer by several degrees per ion inertial length, the Hall current term becomes important in the steady-state generalized Ohm's law

$\boldsymbol{E}+\frac{1}{c} \boldsymbol{u} \times \boldsymbol{B}=\frac{1}{n e c} \boldsymbol{J} \times \boldsymbol{B}+\eta \boldsymbol{J}$,

Here the first term on the right-hand side is the Hall current, $\eta$ is the resistivity, the gradient of electron pressure is neglected, Gaussian units are used, and

$\boldsymbol{J}=\frac{c}{4 \pi} \nabla \times \boldsymbol{B}$.

Similarly, the rotation of the fluid velocity makes the viscous term an important partner in the equation of motion.

We explored whether the Hall-MHD effect can explain the existence of the observed double discontinuities. Our first attempt is to obtain steady-state solutions based on the HallMHD theory to show the merging of a rotational layer and a slow shock layer to form a steady-state compound structure like the observed double discontinuities (Whang, 2002).

All variables are functions of $x$ only. From the divergence free condition we obtain

$B_{x}=$ const.

We use subscript 1 to denote the flow conditions in the upstream region of the discontinuity. Without loss of generality we can set $\boldsymbol{u}_{\mathbf{1}} \| \boldsymbol{B}_{\mathbf{1}}$, the flow is field-aligned in the upstream 
region (De Hoffman and Teller, 1950; Colburn and Sonett, 1970; Scudder, 1987). In this frame of reference we obtain from Faraday's law of induction

$E_{y}=E_{z}=0$

Now we have

$u_{z} B_{x}-u_{x} B_{z}=\frac{c}{4 \pi}\left(\frac{1}{n e} \frac{d B_{y}}{d x}-\eta c \frac{d B_{z}}{d x}\right)$,

and

$u_{x} B_{y}-u_{y} B_{x}=\frac{c}{4 \pi}\left(\frac{1}{n e} \frac{d B_{z}}{d x}+\eta c \frac{d B_{y}}{d x}\right)$.

The continuity equation and the three components of the equation of motion can be integrated to give

$\rho u_{x}=m=$ const,

$\frac{4}{3} \mu \frac{d u_{x}}{d x}=m u_{x}+P+\frac{B_{t}^{2}}{8 \pi}-\left(m u_{x 1}+P_{1}+\frac{B_{t 1}^{2}}{8 \pi}\right)$,

$$
\mu \frac{d u_{y}}{d x}=m u_{y}-\frac{B_{x} B_{y}}{4 \pi},
$$

and

$\mu \frac{d u_{z}}{d x}=m u_{z}-\frac{B_{x} B_{z}}{4 \pi}$.

It is convenient to introduce a dimensionless variable $\xi=x / \lambda$, a dimensionless resistivity $\mathcal{R}=\eta$ cen $_{1} / B_{x}$, and a dimensionless viscosity $\mathcal{M}=\mu / m \lambda$. In order to show the rotational variations of the magnetic field and the fluid velocity, we introduce

$B_{y}=B_{t} \cos \phi, \quad B_{z}=B_{t} \sin \phi$,

$u_{y}=u_{t} \cos \alpha, \quad u_{z}=u_{t} \sin \alpha$.

Now, we can write the Ohm's law as

$\frac{d}{d \xi}\left(\frac{B_{t}}{B_{x}}\right)-\mathcal{R} \frac{u_{x 1}}{u_{x}} \frac{B_{t}}{B_{x}} \frac{d \phi}{d \xi}=\frac{u_{t}}{u_{x}} \sin (\alpha-\phi)$

and

$\frac{d \phi}{d \xi}+\mathcal{R} \frac{u_{x 1}}{u_{x}} \frac{B_{x}}{B_{t}} \frac{d}{d \xi}\left(\frac{B_{t}}{B_{x}}\right)=1-\frac{u_{t}}{u_{x}} \frac{B_{x}}{B_{t}} \cos (\alpha-\phi)$.

and the three components of the equation of motion as

$\frac{4}{3} m \mathcal{M} \frac{d u_{x}}{d \xi}=m u_{x}+P+\frac{B_{t}^{2}}{8 \pi}-\frac{1+\beta_{1}+\cos ^{2} \theta_{1}}{8 \pi \cos ^{2} \theta_{1}}$,

$m \mathcal{M} \frac{d u_{t}}{d \xi}=m u_{t}-\frac{B_{x} B_{t}}{4 \pi} \cos (\alpha-\phi)$,

and

$m \mathcal{M} \frac{d \alpha}{d \xi}=\frac{B_{x} B_{t}}{4 \pi u_{t}} \sin (\alpha-\phi)$.

When the angle $\alpha /=\phi$, the fluid velocity $\boldsymbol{u}$ is not fieldaligned. In the above equations $\theta_{1}$ is the acute angle between the normal vector and the magnetic field in the upstream and $\beta_{1}=8 \pi P_{1} / B_{1}^{2}$. The effect of Joule heating and viscous dissipation are negligibly small in the energy equation, the flow is practically isentropic inside the rotational layer. We use the isentropic equation

$\frac{d P}{d \rho}=\frac{5}{3} \frac{P}{\rho}$.

The system of governing Eqs. (1)-(7) can be used to study the variations of seven variables $\left(B_{t}, \phi, u_{x}, u_{t}, \alpha, \rho\right.$, and $P$ ) through the rotational discontinuity.

\section{Two-layer Hall-MHD model}

We use a two-layer model to study steady-state Hall-MHD solutions of double discontinuity. The model assumes that a compound structure consists of a slow shock layer followed by a rotational layer; all flow properties are continuous across the interface between the two layers, and $\alpha=\phi=0$ throughout the shock layer. The model also assumes that on the rotational layer side of the interface $d \alpha / d \xi$ and $d \phi / d \xi$ are not zero. Let subscript 1 denote the conditions in the upstream of the shock layer, and subscript $i$ denote conditions at the interface between the two layers. A set of modified RankineHugoniot relations is used to calculate the jump conditions between region 1 and the interface. The Hall-MHD Eqs. (1)(7) are used to calculate the variations of the plasma and magnetic field in the rotational layer in the downstream of the interface.

The plasma and magnetic field have a jump across the slow shock layer between the upstream region 1 and the interface $i$. The jumps in plasma and magnetic fields across the slow shock layer are described by a system of modified Rankine-Hugoniot relations. In addition to Eq. (1), the system consists of

$$
\begin{gathered}
m u_{x i}+P_{i}+\frac{B_{t i}^{2}}{8 \pi}=m u_{x 1}+P_{1}+\frac{B_{t 1}^{2}}{8 \pi} \\
m u_{t i}-\frac{B_{x} B_{t i}}{4 \pi}=m u_{t 1}-\frac{B_{x} B_{t 1}}{4 \pi}, \\
u_{x i} B_{t i}\left[1-\frac{1}{A}\left\{\left(\mathcal{R} \frac{n_{i}}{n_{1}}\right)^{2}+1\right\}\left(\frac{d \phi}{d \xi}\right)_{i}\right]-B_{x} u_{t i} \\
=u_{x 1} B_{t 1}-B_{x} u_{t 1},
\end{gathered}
$$

and

$\frac{u_{i}^{2}}{2}+\frac{5}{2} \frac{P_{i}}{\rho_{i}}=\frac{u_{1}^{2}}{2}+\frac{5}{2} \frac{P_{1}}{\rho_{1}}$.

Here $A=\sqrt{4 \pi \rho_{1}} u_{x 1} / B_{x}$ is the shock Alfvén number. When $(d \phi / d \xi)_{i}=0$, this system reduces to the classical RankineHugoniot relations. In the absence of the Hall-MHD terms the dimensionless solutions of the classical RankineHugoniot relations for MHD shocks can be expressed as functions of three dimensionless parameter in the preshock region: the shock Alfvén number $A$, the shock angle $\theta$, and the plasma $\beta$ ratio. The solutions of the modified Rankine-Hugoniot relations depend on one more dimensionless variables: the gradient of rotational angle at the interface $(d \phi / d \xi)_{i}$. 

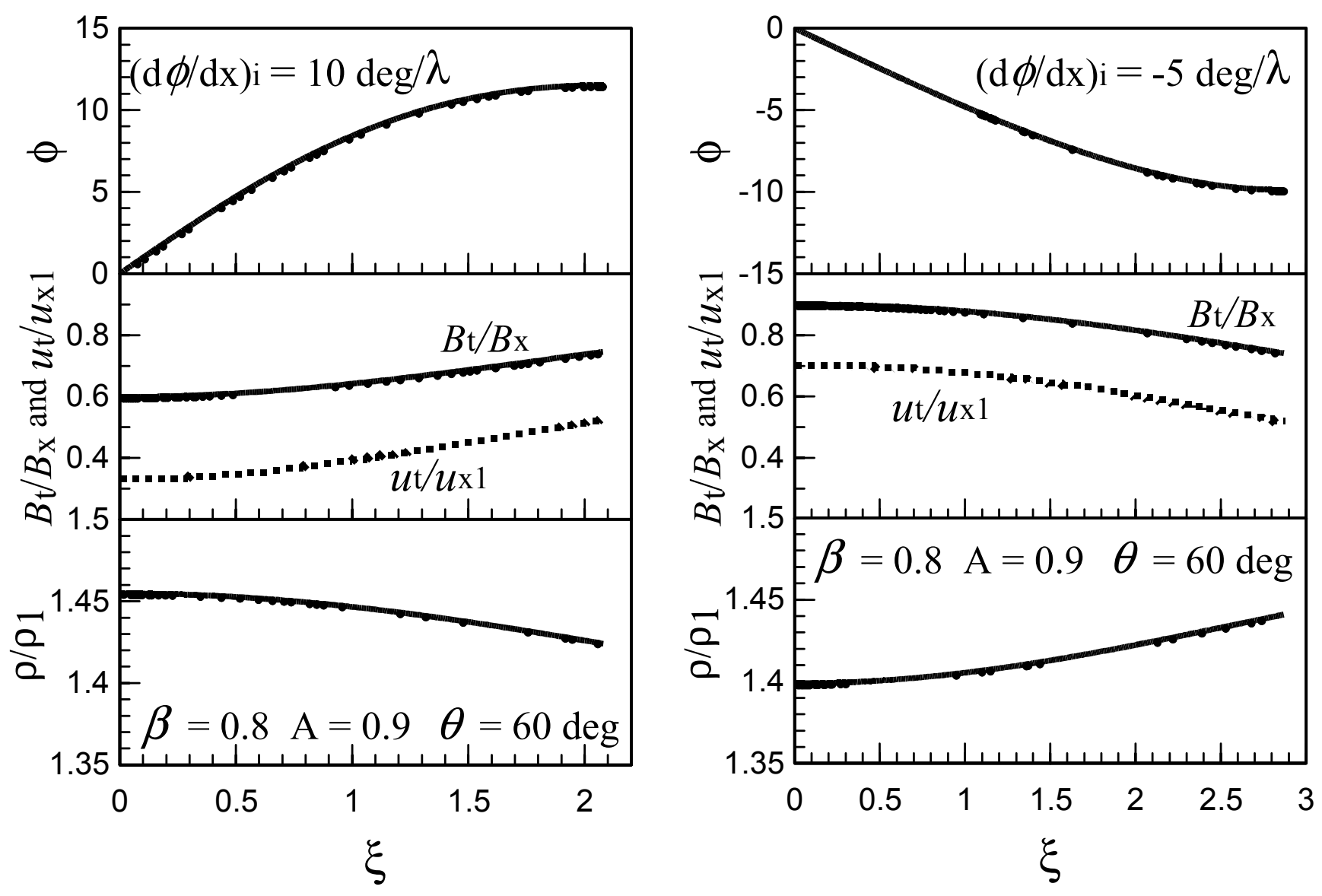

Fig. 6. Two example solutions with $(d \phi / d x)_{i}=10^{\circ} / \lambda$ and $-5^{\circ} / \lambda, \beta=0.8, A=0.9$, and $\theta=60^{\circ}$. The thickness of the rotational layers is about 2 to 3 ion inertial lengths.

We carry out solutions for steady-state model of double discontinuities in two steps. We first calculate the jump conditions between region 1 and the interface using the modified Rankine-Hugoniot relations. Then using the shock solutions at the interface as the boundary condition we solve the HallMHD Eqs. (1)-(7) for the variations of the plasma and magnetic field in the rotational layer in the downstream of the interface.

Figure 6 shows two example solutions. The interface is located at $\xi=0$. The plots for the rotational angle $\phi$ show that the thickness of the rotational layers is about 2 to 3 ion inertial lengths. The flow is compressible; $u_{x}, u_{t}, B_{t}, P$, and $\rho$ change slightly through the rotational layer. The variations in rotational angles $\phi$, the dimensionless tangential velocity and magnetic field $u_{t} / u_{x 1}$ and $B_{t} / B_{x}$ through the rotational layer are proportional to the magnitude of $d \phi / d \xi$ at the interface. The numerical solutions in Fig. 7 show that the thickness of the rotational layer increases at increasing $\beta$. The solutions in Fig. 8 show that the thickness of the rotational layer increases at decreasing $\theta$. The profile of the rotational angle is almost independent of the shock Alfvén number $A$.

\section{Summary and discussion}

A MHD shock has a finite thickness across which physical properties change continuously. We used high-resolution data to examine the magnetic field structure of slow shocks, and found that a slow shock is often followed by an adjoining rotational discontinuity layer on the postshock side. Such a compound structure is known as a double discontinuity. The thickness of the rotational layer is of the order of a few ion inertial lengths; the magnetic field rotates by several degrees per ion inertial length inside the rotational layer.

The high-resolution magnetic field data obtained from Wind and Geotail spacecraft play a vital role in the observation of double discontinuities. The best available time resolutions of the magnetic field are $92 \mathrm{~ms}$ for the Wind data and $62.5 \mathrm{~ms}$ for the Geotail data. Prior to our discovery of double discontinuities, Song et al. (1992) have reported the observation of a possible superposition of a rotational discontinuity and slow mode discontinuity in front of the magnetopause; Seon et al. (1996) have described variations of magnetic fields that could be considered as circularly polarized structures in the shock layer in some slow shock crossings in the distant magnetotail. If better resolution data were 


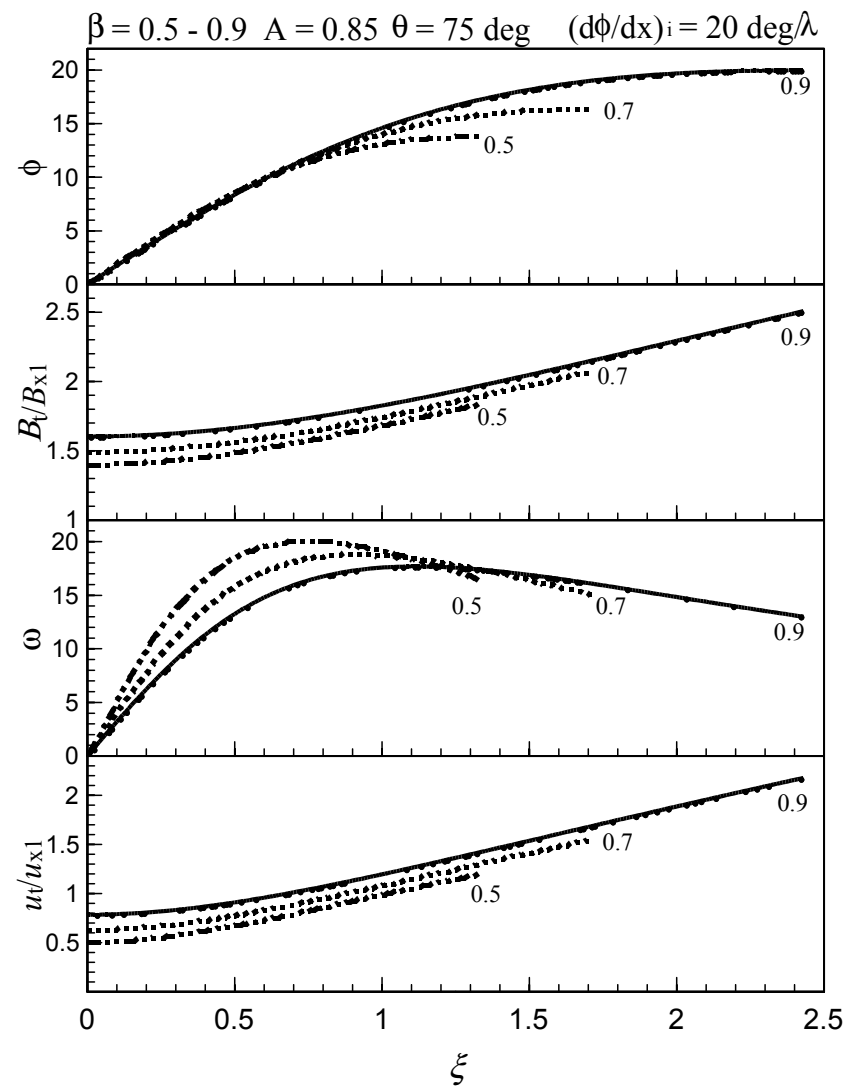

Fig. 7. The thickness of the rotational layer increases at increasing plasma $\beta$ ratio.

used in the analyses, their researches may have been early observations of double discontinuities.

We interpret the formation of double discontinuity as resulting from the interaction of a rotational discontinuity approaching a slow shock from the postshock side; under certain circumstances, the rotational discontinuity cannot penetrate through the slow shock and the two discontinuities merge to form a new compound structure. According to ideal MHD theory, when a rotational discontinuity approaches a slow shock from the postshock side, the rotational discontinuity would always penetrate through the slow shock from behind and then continue to propagate away from the slow shock as a separate MHD discontinuity in the preshock region. Thus, the ideal MHD theory cannot explain why the rotational discontinuity may not penetrate through the shock layer, and the merging of the two layers into a stable compound structure. When the magnetic field rotates by several degrees per ion inertial length inside a thin rotational discontinuity, the Hall current term becomes important in the generalized Ohm's law. We made some preliminary progress to show that steady-state Hall-MHD equations can produce solutions to show the merging of a rotational layer with a slow shock layer to form a compound structure just like the observed double discontinuities.

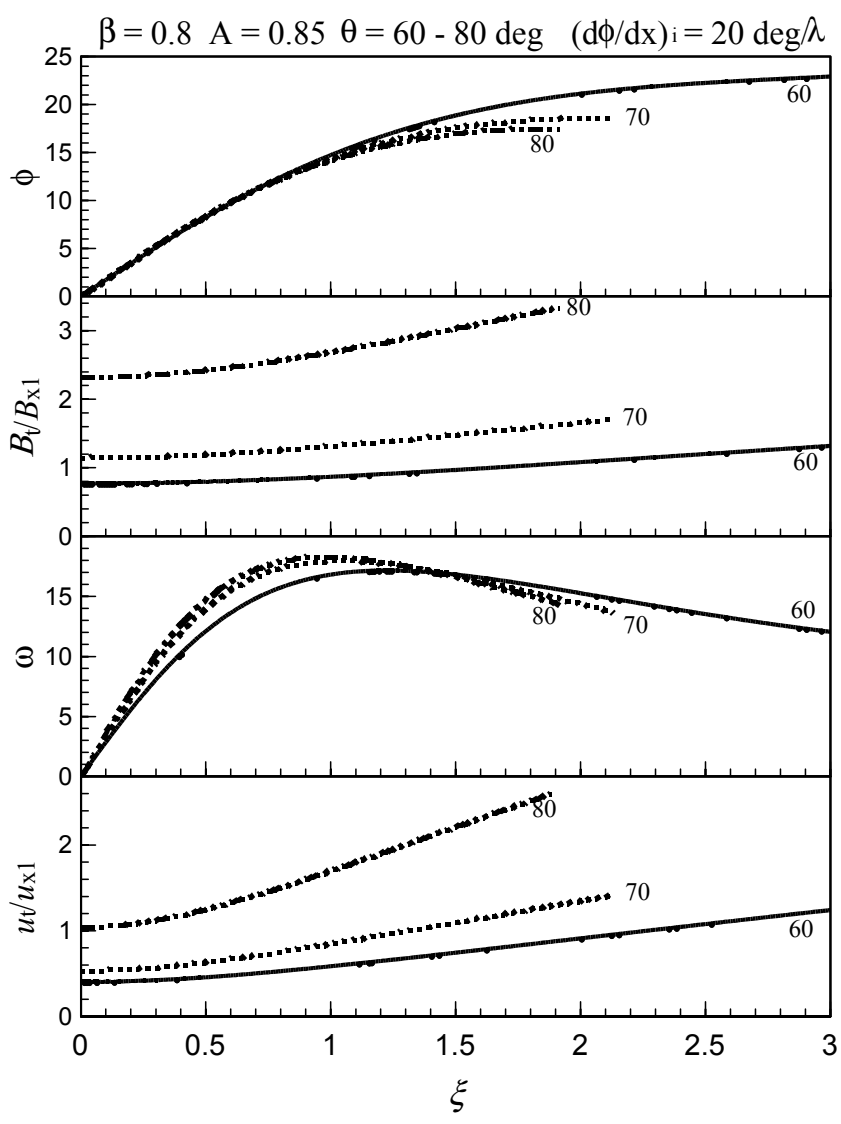

Fig. 8. The thickness of the rotational layer increases at decreasing shock angle $\theta$.

Acknowledgements. The author thanks R. P. Lepping for Wind magnetic field data, S. Kokubun for Geotail magnetic field data, K. W. Ogilvie and A. J. Lazarus for Wind plasma data, and T. Mukai for Geotail plasma data. The work at the Catholic University of America was supported by Department of Energy Grant DE-FG02-00ER54582 and by NSF Grant ATM-0244315.

Edited by: A. S. Sharma

Reviewed by: two referees

\section{References}

Burlaga, L. F. and Chao, J. K.: Reverse and forward slow shocks in the solar wind, J. Geophys. Res., 76, 7516-7521, 1971.

Chao, J. K. and Olbert, S.: Observation of slow shocks in interplanetary space, J. Geophys. Res., 75, 6394-6397, 1970.

Colburn, D. S. and Sonett, C. P.: Discontinuity in the solar wind, Space Sci. Rev., 5, 439-506, 1966.

De Hoffman, F. and Teller, E.: Magneto-hydrodynamic shocks, Phys. Rev. 80, 692-703, 1950.

Feldman, W. C., Baker, D. N., Bame, S. J., Birn, J., Gosling, J. T., Hones Jr., E. W., and Schwartz, S. J.: Slow mode shocks: A semipermanent feature of the distant geomagnetic tail, J. Geophys. Res., 90, 233-240, 1985.

Feldman, W. C., Schwartz, S. J., Bame, S. J., Baker, D. N., Birn, J., Gosling, J. T., Hones Jr., E. W., McComas, D. H., Slavin, J. A., 
Smith, E. J., and Zwickl, R. D.: Evidence for slow-mode shocks in the deep geomagnetic tail, Geophys. Res. Lett., 11, 599-602, 1984.

Feldman, W. C., Tokar, R. L., Birn, J., Hones Jr., E. W., Bame, S. J., and Russell, C. T.: Structure of a slow mode shock observed in plasma sheet boundary layer, J. Geophys. Res., 92, 83-94, 1987.

Lee, L. C., Wu, B. H., Chao, J. K., Lin, C. H., and Li, Y.: Formation of a compound slow shock-rotational discontinuity structure, J. Geophys. Res., 105, 13 045-13 053, 2000.

Richter, A. K., Rosenbauer, H., Neubauer, F. M., and Ptitsyna, N. G.: Solar wind observations associated with a slowforward shock wave at 0.31 AU, J. Geophys. Res., 90, 75817586, 1985.

Saito, Y., Mukai, T., Terasawa, T., Nishida, A., Machida, S., Hirahara, M., Maezzawa, K., and Yamamoto, T.: Slow-mode shocks in the magnetotail, J. Geophys. Res., 100, 23 567-23 581, 1995.

Saito, Y., Mukai, T., Terasawa, T., Nishida, A., Machida, S., Kokubun, S., and Yamamoto, T.: Foreshock structure of the slow-mode shocks in the Earth's magnetotail, J. Geophys. Res., 101, 13 267-13 274, 1996.

Scudder, J. D.: The field-aligned flow approximation for electrons within layers possessing a normal mass flux: a corollary to the de-Hoffmann-Teller theorem, J. Geophys. Res., 92, $13447-$ 13 455, 1987.

Seon, J., Frank, L. A., Paterson, W. R., Scudder, J. D., Coroniti, F. V., Kokubun, S., and Yamamoto, T.: Observations of a slow-mode shock at the lobe-plasma sheet boundary in Earth's distance magnetotail, Geophys. Res. Lett., 22, 2981-2984, 1995.
Seon, J., Frank, A., Paterson, W. R., Scudder, J. D., Coroniti, F. V., Kokubun, S., and Yamamoto, T.: Observations of slow-mode shocks in Earth's distant magnetotail with the Geotail spacecraft, J. Geophys. Res., 12, 27 383-27 398, 1996.

Smith, E. J., Slavin, J. A., Tsurutani, B. T., Feldman, W. C., and Bame, S. J: Slow mode shocks in the earth's magnetotail: ISEE 3, Geophys. Res. Lett., 11, 1054-1057, 1984.

Song, P., Russell, C. T., and Thomsen, M. F.: Slow mode transition in the frontside magnetosheath, J. Geophys. Res., 97, 82958305, 1992.

Whang, Y. C.: Hall magnetohydrodynamics model of double discontinuities, Phys. Plasmas, 9, 4905-4910, 2002.

Whang, Y. C., Fairfield, D., Lepping, R. P., Mukai, T., Saito, Y., Slavin, J., and Szabo, A.: Double discontinuities at the magnetotail plasma sheet-lobe boundary, Ann. Geophys., 19, 1095-1105, 2001.

Whang, Y. C., Fairfield, D., Smith, E. J., Lepping, R. P., Kokubun, S., and Saito, Y.: Observations of double discontinuities in the magnetotail, Geophys. Res. Lett., 24, 3153-3156, 1997.

Whang, Y. C., Zhou, J., Lepping, R. P., and Ogilvie, K. W.: Interplanetary slow shock observed from Wind, Geophys. Res. Lett., 23, 1239-1242, 1996.

Whang, Y. C., Zhou, J., Lepping, R. P., Szabo, A., Fairfield, D., Kokubun, S., Ogilvie, K. W., and Fitzenreiter, R.: Double discontinuity: A compound structure of slow shock and rotational discontinuity, J. Geophys. Res., 103, 6513-6520, 1998. 